Hans-Erlwein-Gymnasium Dresden

Komplexe Leistung

\title{
Mögliche Auswirkungen des Brexits auf die britische Finanzbranche am Beispiel der City of London
}

Verfasser:

Kurs:

Tutor:

Betreuer:

Abgabetermin:
Paula Eisoldt

11DE1

Herr Wiegand Herr Wiegand

04.03.2019 


\section{Inhaltsverzeichnis}

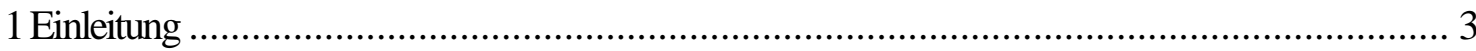

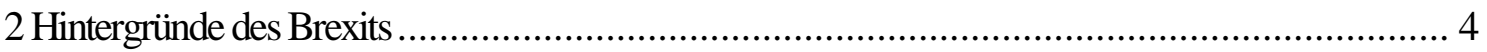

3 Wirtschaftliche Auswirkungen auf das europäische Bankensystem........................................ 7

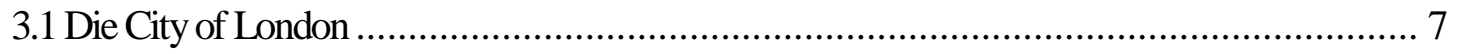

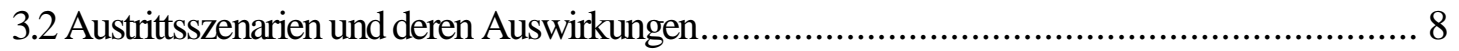

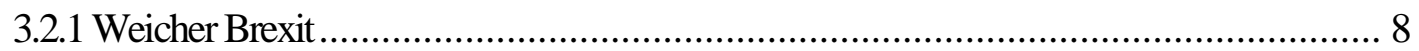

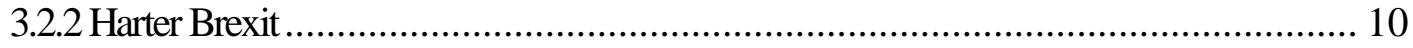

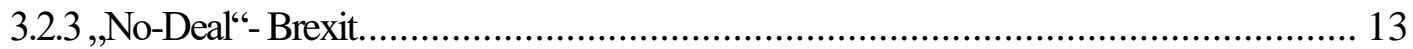

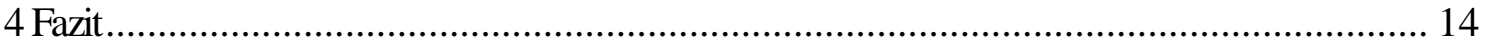

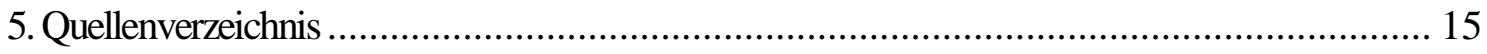

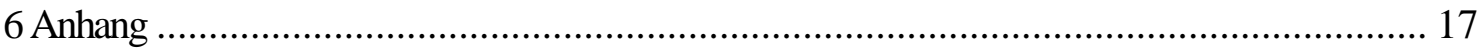




\section{Einleitung}

Am 23. Juni 2016 fand in Großbritannien das Referendum über den Verbleib des Landes in der Europäischen Union statt. ${ }^{1}$ An der Abstimmung beteiligten sich 71,8 Prozent $^{2}$ der insgesamt 46,5 Millionen wahlberechtigten Bürger des Vereinigten Königreichs ${ }^{3}$.

Das Ergebnis überraschte viele: Insgesamt 51,9 Prozent der Menschen stimmten an diesem Tag für den Austritt aus der EU und nur 48,1 Prozent dagegen. ${ }^{4}$

Mit Blick auf die britische Verfassung ist ein solches Votum rechtlich nicht bindend. Es bedarf immer noch der Zustimmung der Regierung und des Parlaments, um den Austritt Großbritanniens zu vollziehen. Beide stimmten dem Austritt aus der EU, dem sogenannten Brexit, zu.

Das Kunstwort „Brexit“ ist eine Zusammensetzung der englische Begriffe „Britian“ und „exit", welche aufDeutsch „Britannien“ beziehungsweise „Ausgang“ bedeuten.

Großbritannien wird die EU voraussichtlich am 29. März 2019 verlassen und somit jegliche Rechte eines Mitgliedstaates verlieren. Dies sorgt innerhalb des Landes für große Unsicherheit, da noch nicht feststeht, wie eine zukünftige Beziehung zur EU aussehen wird.

Gerade die Akteure der britischen Wirtschaft sehen dem Brexit deswegen besorgt entgegen und müssen sich derzeit auf mögliche Folgen und Risiken vorbereiten.

Auch die Finanzbranche des Vereinigten Königreichs, welche die wirtschaftliche Führungsrolle im Land einnimmt, ist davon betroffen. Was würde der Brexit für sie bedeuten?

Großbritannien hat nach dem Austritt nicht länger Zugang zum europäischen Markt, was für die Banken katastrophal wäre. Somit werden sich viele Finanzinstitute gezwungen sehen, das Land zu verlassen und einen neuen Standort innerhalb der EU zu eröffnen.

Diese Problematik soll im Hinblick auf verschiedene Austrittsszenarien am Bespiel des britischen Finanzzentrums „City of London“ genauer untersucht werden, denn die Akteure des Finanzsektors versuchten sogar das Referendum im Vorfeld zu verhindern.

\footnotetext{
${ }^{1}$ Vgl. S.17: Anhang 1

${ }^{2}$ https://www.bbc.com/news/uk-politics-32810887 20.02.19

${ }^{3}$ Vgl. König, Johann-Günther: Die spinnen, die Briten. Das Buch zum Brexit. 2. Auflage, Reinbek bei Hamburg: Rowohlt Taschenbuch Verlag, 2016, S.16.

${ }^{4}$ Vgl. Die spinnen, die Briten, S.17.
} 


\section{Hintergründe des Brexits}

Eine Abgrenzung Großbritanniens von den übrigen Mitgliedsstaaten der Europäischen Union wird bereits auf den ersten Blick sichtbar. Im Vereinigten Königreich wird nicht mit Euro, sondern mit Pfund bezahlt, die Autos fahren auf der linken und nicht auf der rechten Straßenseite und gemessen wird bei dem Volumen in Pints statt in Liter.

Generell herrscht ein eher anti-europäisches Denken unter den Bürgern, welches nun durch den „Brexit“ deutlich wurde. Aber wo liegen die Ursachen dafür?

Zwei Drittel der britischen Bürger können sich, trotz der Tatsache, dass Großbritannien in seinen ehemaligen Kolonien keinerlei Macht mehr besitzt, besser mit dem Commonwealth als mit der EU identifizieren. ${ }^{5}$ Viele sind der festen Überzeugung, außerhalb Europas, beispielsweise in Kanada, Australien oder Indien, einen weitaus größeren Einfluss zu besitzen. Dies kann unter anderem auf die in letzten Jahren steigenden Exportanteile im Commonwealth zurückgeführt werden. ${ }^{6}$

Dabei war es der ehemalige britische Premierminister Winston Churchill, der 1946 die Gründung der „Vereinigten Staaten von Europa“ stark befürwortete. Allerdings merkte er ebenfalls an, dass „Wann immer wir zwischen Europa und dem offenen Meer wählen müssten, sollen wir uns immer für das offene Meer entscheiden. “7 Churchill strebte lediglich ein freies, zwangloses Bündnis der europäischen Länder an, bei dem keine Souveränität abgegeben werden musste. Zu dieser Zeit, das heißt nach Ende Zweiten Weltkriegs, war sich Großbritannien, als eine der Siegermächte, seiner Stellung als Weltmacht in Hinblick auf Politik und Wirtschaft bewusst. Man war deshalb überzeugt davon, die europäische Integration vorantreiben zu können, ohne selbst Mitglied zu werden. ${ }^{8}$

Durch die Gründung der Europäischen Gemeinschaft für Kohle und Stahl (EGKS) 1951 erlebten deren Mitgliedsstaaten Belgien, Niederlande, Frankreich, Luxemburg, Italien und die Bundesrepublik Deutschland einen enormen ökonomischen Aufstieg, während sich in der britischen Wirtschaft das komplette Gegenteil wiederspiegelte und sie stagnierte. Dies war einer der Gründe, weshalb Großbritannien an der EGKS starke Kritik übte. ${ }^{9}$

Um nicht vollends von den anderen europäischen Nationen überholt $\mathrm{zu}$ werden, versuchte die damalige Regierung um Premierminister Harold MacMillian die Gründung der Europäischen Wirtschaftsgemeinschaft (EWG), eine Art Weiterentwicklung der EGKS mit den gleichen

${ }^{5} \mathrm{Vgl}$. https://www.merkur.de/politik/was-ist-brexit-warum-brexit-antworten-auf-wichtigsten-fragen-zr-6515881.html 16.09 .18

${ }^{6}$ Vgl. Welfens, Paul J.J.: BREXIT aus Versehen. Europäische Union zwischen Desintegration und neuer EU. 2. Auflage, Springer Fachmedien Wiesbaden GmbH, 2018, S.98.

${ }^{7}$ https://www.welt.de/politik/ausland/article160310873/Warum-die-Briten-Europa-einfach-nicht-moegen.html 16.09.18

${ }^{8}$ Busch, Klaus: Das Versagen Europas: Die Euro- und die Flüchtlingskrise sowie die „Brexit"-Diskussion. Eine Flugschrift. Hamburg: VSA-Verlag, 2016, S.73.

${ }^{9}$ Vgl. Das Versagen Europas, S.73. 
Gründungsstaaten, $1957 \mathrm{zu}$ verhindern, was allerdings misslang. Man sah diese als „Kontinentalsperre“ an, weil sich die Mitgliedsstaaten auf einen gemeinsamen einheitlichen Markt einigten.

1961 stellte dann auch Großbritannien einen Aufnahmeantrag in die spätere Europäische Gemeinschaft, weil die Regierung die wirtschaftlichen Vorteile erkannte und bemerkte, wie schädlich es wäre, kein Mitglied zu sein. Allerdings wurde die Mitgliedschaft seitens der EWG abgelehnt. Der damalige französische Präsident Charles de Gaulle sprach sich gegen das Vereinigte Königreich aus, weil er in ihm ein „trojanisches Pferd“ der USA sah, das sich in die europäische Wirtschaft einmischen wollte, um nicht an Macht und Einfluss zu verlieren. ${ }^{10}$ Letztendlich bewilligte man eine Mitgliedschaft des Vereinigten Königreichs erst 1973. Viele Briten fühlten sich allerdings zu diesem Zeitpunkt bereits ausgegrenzt und betrachteten die Europäische Gemeinschaft kritisch.

Im Jahr nach dem Beitritt hatte Großbritannien große Zahlungsbilanzprobleme und musste einen Kredit von 3,9 Milliarden Dollar aus den Internationalen Währungsfonds aufnehmen. 1986 gelang der damaligen EU-kritischen Premierministerin Margaret Thatcher die Stärkung des nationalen Finanzmarktes. Unter ihrem Nachfolger John Major, welcher wiederrum als Befürworter der EU galt, verlor der Pfund wieder an Wert. Eine Tatsache die viele britische Bürger dazu bewegte, die europäische Staatengemeinschaft als wirtschaftsschädigend $\mathrm{zu}$ betrachten. ${ }^{11}$ Margaret Thatcher entwickelte sich zu einer Ikone aller EU-Gegner und die „Europa-Feindschaft zu einem Zeichen der Treue $^{6.12}$

1987 unterstütze die britische Regierung die Einführung der Einheitlichen Europäischen Akte (EEA) stark. Diese bedeutete zwar einen einheitlichen Binnenmarkt, aber keine Währungsunion und keinen europäischen Bundesstaat. Man musste also kaum Verpflichtungen gegenüber den anderen Mitgliedsstaaten eingehen. Da Großbritannien eben diese Verpflichtungen stets ablehnte, ist es bis heute nicht der europäischen Währungs- und Wirtschaftsunion beigetreten. ${ }^{13}$ Das Vereinigte Königreich ist außerdem kein vollwertiges Mitglied des Schengen-Raums. ${ }^{14}$

Die Abneigung der Briten gegenüber der europäischen Union ist zudem darauf zurückzuführen, dass das Land in der Vergangenheit in gewisser Hinsicht übergangen wurde. So gab es der Geschichte der EU nur einen britischen Kommissionspräsidenten, Roy Jenkins von 1977 bis 1981, während

\footnotetext{
${ }^{10}$ Vgl. S.4: Das Versagen Europas, S.73.

${ }^{11}$ Vgl. S.4: BREXIT aus Versehen, S.98.

${ }^{12}$ Vgl. S.4: www.welt.de/politik

${ }^{13}$ Vgl. S.4: Das Versagen Europas, S.74.

${ }^{14}$ Das Land arbeitet zwar polizeilich und justiziell mit den anderen EU-Staaten zusammen, aber auch EU-Bürger müssen sich bei der Ein- und Ausreise Grenzkontrollen unterziehen.
} 
beispielsweise Frankreich sieben Präsidenten stellte. Außerdem bemühte man sich kaum darum, wichtige EU-Institutionen in Großbritannien anzusiedeln. ${ }^{15}$

Dazu kommt, dass jeder fünfte Brite unterhalb der Armutsgrenze lebt, die Zahl ist tendenziell steigend. ${ }^{16}$ Die Verantwortung hierfür wird bei der EU gesucht, obwohl beispielsweise die Kürzung von Sozialleistungen in den letzten Jahren vielmehr mit der strikten Sparpolitik David Camerons in Verbindung zu bringen ist.

In den letzten Jahren beklagten sich sowohl die Bürger als auch die Regierung des Vereinigten Königreichs über die zunehmende Zahl an Migranten vor allem aus den osteuropäischen EU-Ländern. Man sei mit der jährlichen Zuwanderungszahl von 300000 Menschen überfordert, auch wenn das nur ein Bevölkerungswachstum von 0,4 Prozent bedeutet. ${ }^{17}$

Zudem ging die Flüchtlingskrise nicht spurlos an Großbritannien vorbei und war ein ausschlaggebender Punkt bei dem Brexit-Referendum. Im Vergleich zu anderen europäischen Ländern nimmt Großbritannien zwar nur sehr wenige Asylbewerber auf, aber da auch hier ein rechtspopulistischer Ruck durch die Gesellschaft geht, entwickelten viele Briten eine Abneigung gegenüber den von der EU zugewiesenen Flüchtlingen.

Insgesamt hat sich das anti-europäische Denken Großbritanniens über die letzten Jahrzehnte hinweg kontinuierlich entwickelt. Die Ursachen für Missstände im Land wurden oft nicht bei der eigenen Regierung gesucht, sondern bei der Europäischen Union. Auf der anderen Seite wollte man stets von allen möglichen Vorteilen der Staatengemeinschaft profitieren.

\footnotetext{
${ }^{15}$ Vgl. S.4: BREXIT aus Versehen, S. 104.

${ }^{16}$ Vgl. S.3: Die spinnen, die Briten, S. 87.

${ }^{17}$ Vgl. S.4: BREXIT aus Versehen, S. 106-107.
} 


\section{Wirtschaftliche Auswirkungen auf das europäische Bankensystem}

\subsection{Die City of London}

Zentral in der britischen Hauptstadt gelegen befindet sich der größte und wichtigste europäische

Banken- und Finanzdienstleistungsstandort: die sogenannte City of London.

Es handelt sich dabei nicht um einen der 32 Stadtbezirke Londons, sondern um ein exterritoriales Gebiet in der Stadt. Damit unterliegt dieses ungefähr 2,6 Quadratkilometer große Areal nicht der britischen Regierung oder Londons Bürgermeister, ${ }^{18}$ sondern die City richtet sich nach ihren eigenen Gesetzen und wird von einer eigenen Art Regierung, der City of London Corporation, geführt und überwacht.

Die im Jahr 1067 gegründete Corporation ist die älteste Kommunalverwaltung der Welt. ${ }^{19}$ Dessen Vorsitzender und Repräsentant ist der Lord Major of London, welcher jeweils für ein Jahr gewählt wird. Wahlberechtigt sind alle 8.000 Einwohner mit jeweils einer Stimme und die angesiedelten Unternehmen mit insgesamt 23.000 Stimmen, jeweils eine Stimme für jeden Mitarbeiter. ${ }^{20}$

Die City of London, auch „Square Mile“ genannt, ist nicht nur Standort britischer Unternehmen, sondern hier befinden sich außerdem insgesamt 241 ausländische Banken. ${ }^{21}$ Der Vorteil für Banken ist, dass von der City aus relativ einfach weltweit mit Wertpapieren und Devisen gehandelt werden kann, denn die Regulierungen seitens Großbritanniens oder der EU-Kommission greifen hier nicht. Die Corporation ist für die Beaufsichtigung zuständig, allerdings besteht die Regierung hauptsächlich aus Bankern und Unternehmern, welche nach Profit streben und sehr unternehmerfreundlich agieren. So steht die City of London oft wegen angeblicher Korruption und dem Verdacht auf Lenkung von Steuerhinterziehung in der Kritik. ${ }^{22}$

Deutlich wird die Unabhängigkeit der City auch im Hinblick auf die Geschichte. Nachdem das Pfund Sterling 1956 als Leitwährung im Welthandel bedroht war, ${ }^{23}$ verhängte die britische Regierung ein Verbot über die Vergabe von Auslandkrediten, um die eigene Wirtschaft und die Währung zu schützen. Die City of London hatte bis dato auch in Pfund Sterling gehandelt, wollte aber durch die neue Regelung Großbritanniens nicht die Vormachtstellung im Welthandel verlieren. Ab sofort

\footnotetext{
${ }^{18}$ Vgl. S.3: Die spinnen, die Briten, S.82.

${ }^{19}$ Vgl. http://www.faz.net/aktuell/wirtschaft/wirtschaftspolitik/die-city-ringt-mit-dem-brexit-14091163.html 16.10.18

${ }^{20} \mathrm{Vgl}$. https://www.wissensmanufaktur.net/city-of-london/ 15.10.18

${ }^{21}$ Vgl. S.7: www.wissensmanufaktur.net

${ }^{22}$ Vgl. S.3: Die spinnen, die Briten, S.85.

${ }^{23}$ Grund war der Konflikt mit Ägypten über den Suez-Kanal.
} 
wurden Auslandskredite in Dollar vergeben. ${ }^{24}$ Seitdem gilt das Finanzzentrum als Dollarmarkt Europas. $^{25}$

Beliebt ist die City of London als Hauptniederlassung vor allem bei Banken aus Amerika oder Asien, weil sie hier den sogenannten ,financial passport“, also den Finanzpass, erhalten. Dieser ermöglicht es im gesamten Europäischen Wirtschaftsraum, das heißt auch in den Nicht-EU-Ländern wie Norwegen, Island und Liechtenstein, Dienstleistungen anzubieten ohne einen zusätzlichen Standort im jeweiligen Mitgliedsstaat zu besitzen. Zudem obliegt die Beaufsichtigung der Banken den Behörden im Land der Hauptniederlassung, in diesem Falle bei der City of London Corporation, und das Handeln wird nicht zusätzlich in dem Staat, in dem gehandelt wird, überwacht. ${ }^{26}$

Derzeit hat das Vereinigte Königreich einen Marktanteil von 90 Prozent an allen Großhandelsbankenmärkten der EU-Mitgliedsstaaten, ${ }^{27}$ der Großteil davon befindet sich im Finanzsektor Londons.

Zehn Prozent des Bruttoinlandproduktes Großbritanniens werden durch den Finanzdienstleistungssektor erzielt. ${ }^{28}$ Der Handelsüberschuss beim Export ist mit 58 Milliarden Pfund in dieser Branche mit Abstand am höchsten. ${ }^{29}$

\subsection{Austrittsszenarien und deren Auswirkungen}

\subsubsection{Weicher Brexit}

Bisweilen ist noch ungeklärt, wie genau der Austritt Großbritanniens aus der EU ablaufen wird und in welchem Verhältnis die Beteiligten stehen werden.

Eine Möglichkeit ist der sogenannte ,,weiche Brexit“. Hierbei handelt es sich um ein Abkommen, das den Verbleib des Vereinigten Königreichs in der Zollunion und im Binnenmarkt, im Zusammenhang mit einer weiterhin bestehenden Mitgliedschaft im Europäischen Wirtschaftsraum (EWR), vorsieht.

Das würde bedeuten, dass unter anderem die Personenfreizïgigkeit weiterhin gewährleistet ist. ${ }^{30}$

Außerdem wäre durch das Fortbestehen der Mitgliedschaft in der Zollunion und im EWR der freie Handel auf dem EU-Markt durch das Recht auf die EU-Pässe weiter möglich.

\footnotetext{
${ }^{24}$ Laut dem Währungsabkommen von Bretton Woods ist es nur der nationalen Notenbank erlaubt die jeweilige Währung zu drucken und zu vergeben. Weder die Bank of England noch die US-Notenbank konnten Einfluss auf die Geschehnisse in der City nehmen. Vgl. S.7: www.faz.net

${ }^{25} \mathrm{Vgl}$. S.7: www.wissensmanufaktur.net

${ }^{26}$ Vgl. https://bankenverband.de/dossier/brexit/der-brexit-und-die-banken/ 23.09.18

${ }^{27}$ Vgl. S.18: Anhang 2

${ }^{28}$ Vgl. S.3: Die spinnen, die Briten, S.97.

${ }^{29}$ Vgl. S.18: Anhang 3

${ }^{30}$ Vgl. https://www.zeit.de/news/2017-06/12/eu-harter-brexit-weicher-brexit---was-heisst-das-eigentlich-12125404 10.01.19
} 
Ein solches Abkommen würde dem Verhältnis der Europäischen Union mit Norwegen ähneln. Das Land hat vollständigen Zugang zum Binnenmarkt und ist Mitglied des EWR. Es muss im Gegenzug dafür in den EU-Haushalt einzahlen und viele der Gesetzgebungen aus Brüssel akzeptieren und einhalten. Auch Großbritannien müsste im Falle eines ,weichen“ Brexits diese Vorgaben erfüllen. ${ }^{31}$

Genau wie Norwegen hätte das Vereinigte Königreich kein Mitspracherecht bei EU-Beschlüssen, müsste sie aber dennoch einhalten. ${ }^{32}$

Für die City of London hätte ein „weicher" Brexit den Vorteil, dass es wenig bis keine Abwanderung der Banken in andere EU-Länder gäbe. Die Bedingungen wären denen vor dem Austritt sehr ähnlich.

Der europäische Finanzpass würde für die in London ansässigen Institute gelten. ${ }^{33}$

Überdies könnten die Banken die Vorzüge der City nach wie vor genießen. Die Corporation handelt unternehmensfreundlicher als andere Länder es täten, denn in ihr übernehmen die Banker und Unternehmer selbst administrative Aufgaben. ${ }^{34}$ Zudem hat sich das Wirtschaften im Finanzsektor über Jahrzehnte entwickelt und ein Know-how seitens aller Beteiligten ist bereits vorhanden.

Ein weiterer Vorteil für die britische Wirtschaft und die City of London wäre, dass das Pfund aller Voraussicht nach wieder an Wert gewinnen würde. ${ }^{35}$

Nachteilig wäre hingegen die andauernde Bindung an EU-Richtlinien. ${ }^{36}$ Großbritannien verkündete im September 2017, dass eine Bankenderegulierung in Betracht gezogen wird. ${ }^{37}$

Dies würde London für die Finanzinstitute attraktiver gestalten, weil es somit weniger staatliche Eingriffe in das Marktgeschehen gäbe. Jedoch sollte sich dieses Vorhaben schwierig gestalten, wenn sich das Vereinigte Königreich weiterhin an die Vorgaben der Europäischen Union halten muss. Die EU achtet seit der Finanzkrise 2007 sehr genau auf die Bankenregulierung.

Neu wäre nach dem Brexit, dass Großbritannien nicht länger ein Mitspracherecht bezüglich Entscheidungen in Brüssel hätte, welche den Finanzsektor betreffen würden.

Bei den „Brexiteers ${ }^{638}$ trifft der ,weiche Brexit““ auf wenig Zuspruch. Sie sehen darin keine Unabhängigkeit Großbritanniens, sondern vielmehr eine andauernde Mitgliedschaft, diesmal

\footnotetext{
${ }^{31} \mathrm{Vgl}$. https://www.bpb.de/internationales/europa/brexit/229499/folgen-fuer-den-finanzsektor 12.01.19

${ }^{32} \mathrm{Vgl}$. https://www.sueddeutsche.de/wirtschaft/eu-austritt-norwegen-warnt-die-briten-vor-brexit-1.2985562 12.01.19

${ }^{33} \mathrm{Vgl}$. S.8: 3.1 Die City of London

${ }^{34}$ Vgl. S.7: 3.1 City of London

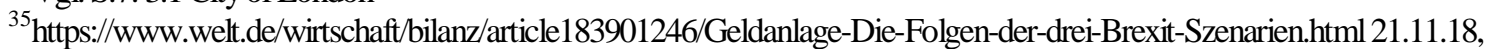

${ }^{36}$ Die Europäische Zentralbank entscheidet über die Erteilung der Banklizenzen und erlässt Anweisungen für alle Banken die in der EU einen Standort besitzen. Finanzinstitute in der EU müssen festgelegte Eigenkapital- und Liquiditätsanforderungen erfuillen, bei Fehlverhalten der Banken kann seitens der Behörden eingegriffen werden und dieses beispielsweise mit Geldstrafen oder der Suspension des Geschäftsleiters sanktioniert werden. Vgl. https://www.bundesfinanzministerium.de/Content/DE/Standardartikel/Themen/Europa/Stabilisierung_des_Euro/Bankenun ion/Bankenaufsicht/einheitliche-bankenaufsicht.html 20.02.19

${ }^{37}$ Vgl. S.4: BREXIT aus Versehen, S.360.

${ }^{38}$ EU-Gegner, welche für den Austritt gestimmt haben
} 
allerdings ohne Rechte. Genau die Punkte, von denen sie sich entlasten wollten, wie beispielsweise die Arbeitnehmerfreizügigkeit, die Beitragszahlungen oder auch die Gesetzesvorgaben, würden weiterhin greifen. $^{39}$

Zudem schloss Premierministerin Theresa May bereits Anfang 2018 eine gemeinsame Zollunion mit der EU aus: „Es ist nicht unsere Politik, in der Zollunion zu sein. Es ist nicht unsere Politik, in [irgend]einer Zollunion zu sein“. ${ }^{40}$ Auch der Verbleib im Binnenmarkt kommt für May nicht in Frage.

\subsubsection{Harter Brexit}

Dem „weichen Brexit“" gegenüber steht der ,harte Brexit“. In diesem Fall des Austritts gäbe es zwar ebenfalls einen Austrittsvertrag und eine politische Erklärung mit grundlegenden Aspekten über das zukünftige Verhältnis zwischen Großbritannien und der Europäischen Union. Aber das Vereinigte Königreich wäre nicht länger Teil des Binnenmarktes, der Zollunion oder dem Europäischen Wirtschaftsraum.

Die geplante Übergangsphase von 2019 bis 2021 würde dann dafür genutzt werden, ein Freihandelsabkommen zu schließen. ${ }^{41}$

Zunächst würde der Austritt aus dem Binnenmarkt und der Zollunion das Ende der Personenfreizïgigkeit zwischen Großbritannien und der EU bedeuten. Auch der Handel wäre durch die damit entstehenden Grenzkontrollen nur noch eingeschränkt möglich, was durch anfallende Zölle noch verstärkt werden würde. Der Warenverkehr zwischen der Europäischen Union und dem Vereinigten Königreich könnte um bis zu 50 Prozent einbrechen. ${ }^{42}$

Das Problem für den Finanzsektor stellt insbesondere der neue Status Großbritanniens als Drittstaat dar. Damit verbunden ist der Verlust des Finanzpasses. ${ }^{43}$

Der Handel mit Finanzdienstleistungen in der EU würde sich komplizierter gestalten, da der Zugang zum europäischen Markt nun erschwert wäre. Die Banken, welche teilweise aus Amerika oder Asien kommen und sich mit einem Tochterunternehmen den Zugang zum europäischen Markt sichern, müssten sich zusätzlichen Kontrollen unterziehen und einen weiteren Standort in einem EU-Land haben. Sie könnten beispielsweise eine zusätzliche Zweigstelle innerhalb der Europäischen Union errichten, was verwaltungstechnisch zwar einen geringeren Aufwand bedeuten würde, somit

\footnotetext{
${ }^{39}$ Sichtweise der Brexiteers auf die Folgen für die City of London: Vgl. S.13: 3.2.2. Harter Brexit

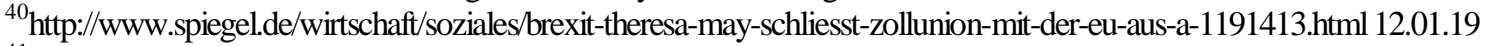

${ }^{41}$ womöglich ähnlich dem CETA-Abkommen mit Kanada, Vgl. Pribyl, Katrin und Drewes, Detlev: Szenarien des Austritts: Mit oder ohne Deal, hart oder weich, in: Trierischer Volksfreund Nr. 240 - Bitburger Zeitung, 16.10.2018, S. 5.

${ }^{42}$ Vgl. Hagen, Norbert: Der Brexit wird vorerst zum zahmen Brino. EU-AUSTRITT GROSSBRITANNIENS Die britische Regierungschefin Theresa May steuert auf eine weiche Trennung von der EU zu - zum Vorteil aller Beteiligten. Wie das Modell bisher aussieht, in: Börse Online, 06.12.2018, S.78.

${ }^{43}$ Somit würde die Niederlassungs- und die Dienstleistungsfreiheit in der EU für die Finanzinstitute in der City of London nicht länger gelten.
} 
allerdings der Handel mit Wertpapierdienstleistungen für Privatanleger auf das eine Land beschränkt wäre. ${ }^{44}$ Außerdem bedeutet eine zusätzliche Niederlassung in jedem Fall höhere Kosten, da neue Büros gemietet beziehungsweise gekauft werden müssten. ${ }^{45}$

Nicht-europäische Finanzinstitute müssten nun beim Wirtschaften drei Grenzen passieren: die des eigenen Landes, die Großbritanniens und letzlich auch die der Europäischen Union.

Für viele erscheint die komplette Umlagerung der Banken aus London in die EU am einfachsten. Kurz nach dem Referendum erwartete die Unternehmensberatung PricewaterhouseCoopers einen Verlust von 100000 Arbeitsplätzen in der City. Internationale Großbanken wie Goldman Sachs, JP Morgan oder auch die Deutsche Bank kündigten außerdem an, ihre Geschäfte zukünftig in anderen Finanzzentren abzuwickeln. ${ }^{46}$

Sollten zu viele Banken ihre Geschäfte auf neue Standorte verlagern, würde London dadurch womöglich seinen Status als eine der größten Finanzmetropolen weltweit verlieren.

Außerdem ist das reale Wirtschaftswachstum des Vereinigten Königreichs in den letzten vier Jahren um mehr als ein Prozent gefallen. ${ }^{47}$ Die Abwanderung der Banken würde dies wahrscheinlich verstärken, da die Finanzbranche einer der wichtigsten Bestandteile in der britischen Wirtschaft ist.

Um die Unternehmen in London zu halten, besteht die Möglichkeit, ein Freihandelsabkommen mit der Europäischen Union abzuschließen. Allerdings gelten diese Abkommen meistens nicht für Finanzdienstleistungen, da es schwierig ist, einen Konsens bei der Regulierung des Wertpapierhandels zu finden. ${ }^{48}$ Diese Problematik wäre gerade bei Verhandlungen zwischen Großbritannien und der EU sehr brisant. ${ }^{49}$

Die angesprochene Abwanderung der Banken aus London ist bereits zu beobachten: Laut der Bankenaufsicht für Finanzdienstleistungen bauen über 45 Finanzinstitute Standorte in Deutschland aus. ${ }^{50}$ Während der Übergangsphase sollen 750 Milliarden Euro nach Frankfurt verlagert werden ${ }^{51}$ und neben der Bank of England auch die Citigroup ihren Handel mit Wertpapieren in Paris

\footnotetext{
${ }^{44} \mathrm{Vgl}$. 2. EU-Richtlinie über Märkte für Finanzinstrumente (MiFID II)

${ }^{45}$ Vgl. Paul, Thomas: Brexit und Banken - Was nun?. Englische Institute müssen kontinentaleuropäische Aktivitäten ebenso neu strukturieren wie Häuser aus der EU im Vereinigten Königreich, in: Börsen-Zeitung, 02.07.2016, S.9.

${ }^{46}$ Vgl. Britische Finanzbranche macht nach Brexit Druck. EU-Marktzugang soll trotz Austritt sichergestellt werden, in: Frankfurter Allgemeine Zeitung, 04.08.2016, S.18.

${ }^{47}$ Vgl. S.19: Anhang 4

${ }^{48}$ Vgl. S.9: www.bpb.de/internationales

${ }^{49}$ Vgl. S.9: 3.2.1 Weicher Brexit

${ }^{50}$ Vgl. https://www.handelsblatt.com/unternehmen/industrie/airbus-ford-sony-die-angst-vor-dem-no-deal-brexit-erfasst-diekonzerne/23907912.html?ticket=ST-4180095-bdTHrdv1V410MhddeOMa-ap5 27.01.19

${ }^{51} \mathrm{Vgl}$. https://www.welt.de/newsticker/bloomberg/article187608500/Banken-verlagern-750-Mrd-wegen-Brexit-nachFrankfurt-Grafik.html 26.01.19
} 
verstärken. ${ }^{52}$ London würde aber voraussichtlich weiterhin ein sehr wichtiges Finanzzentrum bleiben. Viele Institute werden ihren Standort in der City auch trotz eines ,harten Brexits“ nicht komplett aufgeben.

Der Finanzsektor in London verfügt über ein Know-how, mit dem andere europäische Städte nicht mithalten können. Der Handel hat sich hier über Jahrzehnte hinweg entwickelt und kann sich dank der Erfahrungen an neue Gesetzgebungen schnell anpassen. Die JP-Morgan-Deutschland-Chefin Dorothee Blessing sagt in diesem Zusammenhang in einem Interview: „Die Rolle von London kann wohl keine andere Stadt in der EU in absehbarer Zeit übernehmen “. ${ }^{53}$ Dennoch hat JP Morgan bereits Lizenzen in Dublin, Luxemburg und Frankfurt, um nach dem Austritt weiterhin problemlos in der EU handeln zu können.

Nach dem Austritt unterläge der Finanzmarkt in London auch nicht mehr den Regulierungen aus Brüssel. Diese mögliche Deregulierung der Bankenaufsicht wäre vor allem für nicht-europäische Investoren attraktiv.

Auf der anderen Seite sind die EU-Gesetze beim Handeln innerhalb der Europäischen Union, ungeachtet des Standorts, unumgänglich. Wer im Europäischen Wirtschaftsraum handeln möchte, muss sich an die EU-Richtlinien halten, auch im Finanzwesen.

Einer der womöglich größten Verluste, welche die City of London aufgrund des Brexits verkraften müsste, ist die sehr wahrscheinliche Verlagerung der Euro-Clearing Geschäfte. Fast 80 Prozent der Derivategeschäfte in Euro werden durch das Londoner Clearinghaus LCH getätigt. ${ }^{54}$

Die Bank of England spricht von Derivateverträgen im Wert von 38 Billionen Pfund. ${ }^{55}$

$\mathrm{Zu}$ dem Wegfall dieses wichtigen und sehr begehrten Geschäfts kommt, dass die Verlagerung sich sehr schwierig gestalten würde und kostenintensiv wäre.

Während der Übergangsphase bis 2021 darf das Euro-Clearing in London fortgeführt werden, für die Zeit nach dem Austritt beharrt die EU-Kommission allerdings auf einer anderen Lösung. Das Problem wäre, dass der Europäische Gerichtshof dann keine Macht mehr in Großbritannien hätte und damit auch das Bankengeschehen inklusive der Euro-Clearing Geschäfte nicht mehr überwachen könnte.

\footnotetext{
${ }^{52}$ Vgl. Warner, Claudia: Handeln auf Französisch. Paris könnte nach dem London ablösen und der wichtigste Finanzplatz in der Europäischen Brexit Union werden, in: Die Welt, 02.10.2018, S. 17.

${ }^{53} \mathrm{https} / / / \mathrm{www} . w e l t . d e / w i r t s c h a f t / a r t i c l e 165407642 /$ Keine-Stadt-in-der-EU-kann-Londons-Rolle-uebernehmen.html 26.01 .19

${ }^{54}$ Vgl. S.19: Anhang 5

${ }^{55} \mathrm{Vgl}$. https://www.handelsblatt.com/finanzen/banken-versicherungen/nach-brexit-abstimmung-finanzbranche-fuerchteteinen-regulatorischen-flickenteppich/23872390.html?ticket=ST-3852824-IRiQWhBCFYRwQrn4iOrh-ap5 26.01.19
} 
Der Finanzsektor Londons müsste im Falle eines ,harten Brexits“ mit Verlusten rechnen. Die Banken werden etliche Arbeitsplätze in die EU verlagern, die massenhafte Abwanderung, welche prognostiziert wurde, wird aber vermutlich ausbleiben.

Mit dem Euro-Clearing wird die City vorrausichtlich eines ihrer wichtigsten Geschäfte verlieren. Allerdings bleibt abzuwarten, ob das Vereinigte Königreich und die EU in diesem Punkt doch noch eine für London angenehmere Lösung finden.

Die „Brexiteers“" sehen nur im ,harten Brexit" oder auch einem „No-Deal“-Brexit ${ }^{56}$ eine Chance für die City of London. Sie argumentieren unter anderem damit, dass auch die Entscheidung gegen den Euro das Finanzzentrum gestärkt hätte, es wuchs durch die Beibehaltung des Pfund Sterlings um 20 Prozent. ${ }^{57}$ Außerdem könne man, frei von EU-Regulierungen, den Handel mit Amerika, Asien und Afrika besser ausbauen.

\subsection{3,No-Deal'"- Brexit}

Der sogenannte „No-Deal-Brexit“ beschreibt den Brexit ohne Abkommen. Das heißt, am geplanten Austrittsdatum, dem 29. März 2019, würden Großbritannien und die Europäische Union sich nicht über einen Austrittsvertrag geeinigt haben können.

Man spricht in diesem Fall auch von einem ungeordneten Brexit, welcher fatale Folgen haben könnte, da Großbritannien in diesem Moment alle Rechte eines EU-Mitgliedstaates aberkannt bekommen würde und keinerlei Vereinbarungen über das zukünftige Zusammenarbeiten getroffen worden wären. Damit wäre auch kein Wirtschaften zwischen dem Vereinigten Königreich und der EU möglich, da zum Beispiel britische Produkte keine Zulassungen mehr für den europäischen Markt hätten. ${ }^{58}$

Experten gehen davon aus, dass es zu einer steigenden Inflation kommen würde, was wiederum eine Rezession der britischen Wirtschaft bewirken würde. ${ }^{59}$

Die Auswirkungen auf die City of London wären denen des ,,harten Brexits“sehr ähnlich. Allerdings ist damit zu rechnen, dass die Bankenabwanderung in diesem Falle noch einmal steigen würde. Die Wirtschaft in Großbritannien könnte durch einen ungeordneten Austritt um einiges schwächer werden. Ohne Austrittsvertrag verfiele zudem die Übergangsfrist und Großbritannien und die EU ständen sich ohne jegliche Handelsvereinbarungen oder einen gemeinsamen Markt gegenüber.

\footnotetext{
${ }^{56}$ Vgl. S.13: 3.2.3, ,No-Deal“-Brexit

${ }^{57} \mathrm{Vgl}$. https://www.investing.com/news/world-news/city-of-london\%27s-\%27brexiteers\%27-regroup-for-battle-406010 09.02.19

${ }^{58}$ Vgl. Kager, Marianne: Ist ein harter Brexit noch zu vermeiden?, in: Salzburger Nachrichten, 05.09.2018, S.15.

${ }^{59}$ Vgl. S.9: www.welt.de/wirtschaft/bilanz/
} 


\section{$\underline{\text { 4Fazit }}$}

Der ,,weiche“ Brexit wäre für die Finanzbranche am vorteilhaftesten, da der Zugang zum europäischen Markt nicht verloren ginge. Allerdings ist dieses Austrittsszenario, wie bereits erwähnt, sehr unwahrscheinlich $^{60}$ und kann mit Blick auf die wenigen Wochen noch verbleibende Zeit bis zum Austritt nahezu ausgeschlossen werden. Die Möglichkeit eines „harten“ oder sogar ungeordneten Brexits erscheint viel größer. Damit würden die Banken der City of London den Finanzpass verlieren, was die Geschäfte innerhalb der EU erheblich beeinträchtigen würden.

Dennoch geben die wenigsten Banken ihren Standort in London bisher auf, vielmehr eröffnen sie weitere Stellen in EU-Mitgliedsstaaten. ${ }^{61}$ Das bedeutet zwar im ersten Moment einen höheren Aufwand und die Unterhaltung mehrerer Standorte ist zudem kostenintensiver, würde sich aber auf langfristige Sicht lohnen. Die Finanzinstitute könnten weiterhin in der EU handeln und würden den Anschluss an den Weltmarkt dennoch nicht verlieren. Die Möglichkeit besteht, dass vor allem das Handeln mit Nicht-EU-Ländern vereinfacht werden würde, wenn die EU-Regulierungen nicht länger greifen.

Der Brexit kann also durchaus auch als Chance für die City of London betrachtet werden. Es würde auf kurz- und mittelfristige Sicht für die City sicher Probleme geben, die gelöst werden müssten, um weiterhin lukrativ handeln zu können. Allerdings ist die Wirtschaft in Zeiten der Globalisierung dafür bekannt, flexibel auf Veränderungen zu reagieren, so auch die Finanzinstitute in London.

Das derzeitig größte Risiko besteht in einem „No-Deal“-Brexit. Zwar hat Premierministerin Theresa May bereits einen Vertag mit der EU bezüglich zukünftiger Beziehungen ausgehandelt, jedoch lehnten die britischen Abgeordneten den Vertrag am 15. Januar 2019 ab. ${ }^{62}$ Dennoch stimmten sie gegen einen Brexit ohne Einigung über zukünftige Verhältnisse.

Die EU wiederrum behart auf dem Abkommen und schließt Nachverhandlungen weitestgehend aus. Um auch im Falle eines ungeordneten Brexits handlungsfähig zu bleiben, haben sich viele Banken bereits Lizenzen für andere EU-Länder gesichert.

Es ist zu beachten, dass es sich bei dem Großteil der Untersuchungsergebnisse um Vermutungen handelt. Wie sich Großbritanniens Austritt aus der Europäischen Union tatsächlich auf die britische Finanzbranche auswirkt, wird wohl erst in einigen Jahren genauer feststellbar sein.

\footnotetext{
${ }^{60}$ Vgl. S.9-10: 3.2.1 Weicher Brexit

${ }^{61}$ Vgl. S.11-12: 3.2.2 Harter Brexit

${ }^{62} \mathrm{Vgl}$. https://www.sueddeutsche.de/politik/brexit-vertrag-theresa-may-1.4289217 21.02.19
} 


\section{Quellenverzeichnis}

\section{Literatur}

(1) König, Johann-Günther: Die spinnen, die Briten. Das Buch zum Brexit. 2. Auflage, Reinbek bei Hamburg: Rowohlt Taschenbuch Verlag.

(2) Welfens, Paul J.J.: BREXIT aus Versehen. Europäische Union zwischen Desintegration und neuer EU. 2. Auflage, Springer Fachmedien Wiesbaden GmbH, 2018.

(3) Busch, Klaus: Das Versagen Europas: Die Euro- und die Flüchtlingskrise sowie die „Brexit"Diskussion. Eine Flugschrift. Hamburg: VSA-Verlag, 2016.

(4) Pribyl, Katrin und Drewes, Detlev: Szenarien des Austritts: Mit oder ohne Deal, hart oder weich, in: Trierischer Volksfreund Nr. 240 - Bitburger Zeitung vom 16.10.2018, S. 5.

(5) Hagen, Norbert: Der Brexit wird vorerst zum zahmen Brino. EU-AUSTRITT GROSSBRITANNIENS Die britische Regierungschefin Theresa May steuert auf eine weiche Trennung von der EU zu - zum Vorteil aller Beteiligten. Wie das Modell bisher aussieht, in: Börse Online, 06.12.2018, S.78.

(6) Paul, Thomas: Brexit und Banken - Was nun? Englische Institute müssen kontinentaleuropäische Aktivitäten ebenso neu strukturieren wie Häuser aus der EU im Vereinigten Königreich, in: Börsen-Zeitung, 02.07.2016, S.9.

(7) Britische Finanzbranche macht nach Brexit Druck. EU-Marktzugang soll trotz Austritt sichergestellt werden, in: Frankfurter Allgemeine Zeitung, 04.08.2016, S.18.

(8) Warner, Claudia: Handeln auf Französisch. Paris könnte nach dem London ablösen und der wichtigste Finanzplatz in der Europäischen Brexit Union werden, in: Die Welt, 02.10.2018, S. 17.

\section{Internetquellen:}

(9) https://www.bbc.com/news/uk-politics-32810887 20.02.19

(10)https://www.merkur.de/politik/was-ist-brexit-warum-brexit-antworten-auf-wichtigsten-fragen-zr6515881.html 16.09.18

(11)https://www.welt.de/politik/ausland/article160310873/Warum-die-Briten-Europa-einfach-nichtmoegen.html 16.09.18

(12)http://www.faz.net/aktuell/wirtschaft/wirtschaftspolitik/die-city-ringt-mit-dem-brexit14091163.html 16.10.18

(13)https://www.wissensmanufaktur.net/city-of-london/ 15.10 .18

(14) https://bankenverband.de/dossier/brexit/der-brexit-und-die-banken/ 23.09.18

(15)https://www.zeit.de/news/2017-06/12/eu-harter-brexit-weicher-brexit---was-heisst-das-eigentlich1212540410.01 .19

(16)https://www.bpb.de/internationales/europa/brexit/229499/folgen-fuer-den-finanzsektor 12.01.19 
(17)https://www.sueddeutsche.de/wirtschaft/eu-austritt-norwegen-warnt-die-briten-vor-brexit1.298556212 .01 .19

(18)https://www.bundesfinanzministerium.de/Content/DE/Standardartikel/Themen/Europa/Stabilisier ung_des_Euro/Bankenunion/Bankenaufsicht/einheitliche-bankenaufsicht.html 20.02.19

(19)http://www.spiegel.de/wirtschaft/soziales/brexit-theresa-may-schliesst-zollunion-mit-der-eu-aus-a1191413.html 12.01.19

(20)https://www.handelsblatt.com/unternehmen/industrie/airbus-ford-sony-die-angst-vor-dem-nodeal-brexit-erfasst-die-konzerne/23907912.html?ticket=ST-4180095bdTHrdv1V410MhddeOMa-ap5 27.01.19

(21)https://www.welt.de/newsticker/bloomberg/article187608500/Banken-verlagern-750-Mrdwegen-Brexit-nach-Frankfurt-Grafik.html 26.01.19

(22)https://www.handelsblatt.com/finanzen/banken-versicherungen/nach-brexit-abstimmungfinanzbranche-fuerchtet-einen-regulatorischen-flickenteppich/23872390.html?ticket=ST3852824-1RiQWhBCFYRwQm4iOrh-ap5 26.01.19

(23)https://www.investing.com/news/world-news/city-of-london\%27s-\%27brexiteers\%27-regroupfor-battle-406010 09.02.19

(24)https://www.sueddeutsche.de/politik/brexit-vertrag-theresa-may-1.4289217 21.02.19 


\section{Anhang}

\section{Anhang 1: Vertrag über die Europäische Union, Artikel 50}

Quelle: https://dejure.org/gesetze/EU/50.html 17.02.19 11:00 Uhr

\section{EU-Vertrag}

Titel VI - Schlußbestimmungen (Art 47 - 55)

\section{Art. 50}

(1) Jeder Mitgliedstaat kann im Einklang mit seinen verfassungsrechtlichen Vorschriften beschließen, aus der Union auszutreten.

(2) Ein Mitgliedstaat, der auszutreten beschließt, teilt dem Europäischen Rat seine Absicht mit. Auf der Grundlage der Leitlinien des Europăischen Rates handelt die Union mit diesem Staat ein Abkommen über die Einzelheiten des Austritts aus und schließt das Abkommen, wobei der Rahmen für die künftigen Beziehungen dieses Staates zur Union berücksichtigt wird. Das Abkommen wird nach Artikel 218 Absatz 3 des Vertrags über die Arbeitsweise der Europåischen Union ausgehandelt. Es wird vom Rat im Namen der Union geschlossen; der Rat beschließt mit qualifizierter Mehrheit nach Zustimmung des Europäischen Parlaments.

(3) Die Verträge finden auf den betroffenen Staat ab dem Tag des Inkrafttretens des Austrittsabkommens oder andernfalls zwei Jahre nach der in Absatz 2 genannten Mitteilung keine Anwendung mehr, es sei denn, der Europäische Rat beschließt im Einvernehmen mit dem betroffenen Mitgliedstaat einstimmig, diese Frist zu verlăngern.

(4) Für die Zwecke der Absätze 2 und 3 nimmt das Mitglied des Europäischen Rates und des Rates, das den austretenden Mitgliedstaat vertritt, weder an den diesen Mitgliedstaat betreffenden Beratungen noch an der entsprechenden Beschlussfassung des Europäischen Rates oder des Rates teil.

Die qualifizierte Mehrheit bestimmt sich nach Artikel 238 Absatz 3 Buchstabe b des Vertrags über die Arbeitsweise der Europăischen Union.

(5) Ein Staat, der aus der Union ausgetreten ist und erneut Mitglied werden möchte, muss dies nach dem Verfahren des Artikels 49 beantragen. 
Anhang 2: Marktanteile der EU-Staaten an den gesamten europäischen

\section{Großhandelsbankenmärkten, Vor-Brexit-Ausgangssituation}

Quelle: Paul J.J. Welfens: BREXIT aus Versehen. Europäische Union zwischen Desintegration und neuer EU. 2. Auflage, Springer Fachmedien Wiesbaden GmbH, 2018, S.365

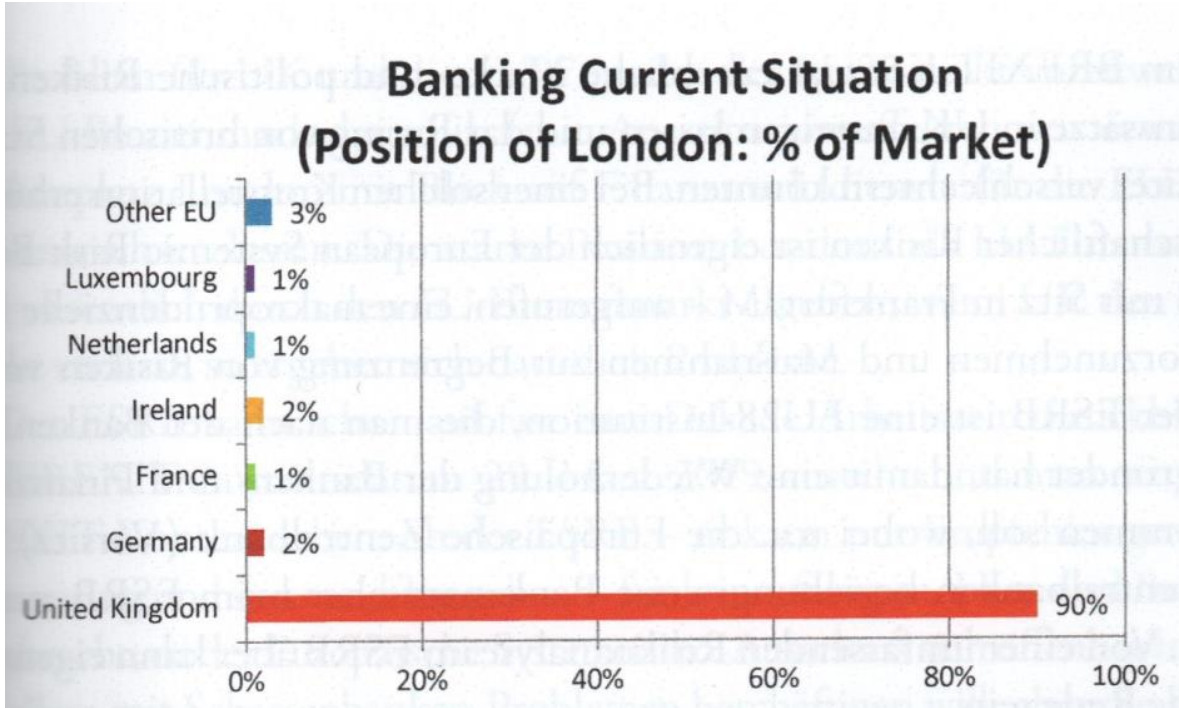

\section{Anhang 3: Die wichtigsten Exportbranchen Großbritanniens}

Quelle: https://www.faz.net/aktuell/finanzen/devisen-rohstoffe/brexit-banken-in-london-wollen-deneu-finanzpass-14472518/infografik-grossbritanniens-14472243.html 22.01.2019 15:40 Uhr

\section{Der Finanzsektor ist die Exportbranche Nr. 1}

Handelsüberschuss britischer Wirtschaftssektoren (Auswahl)

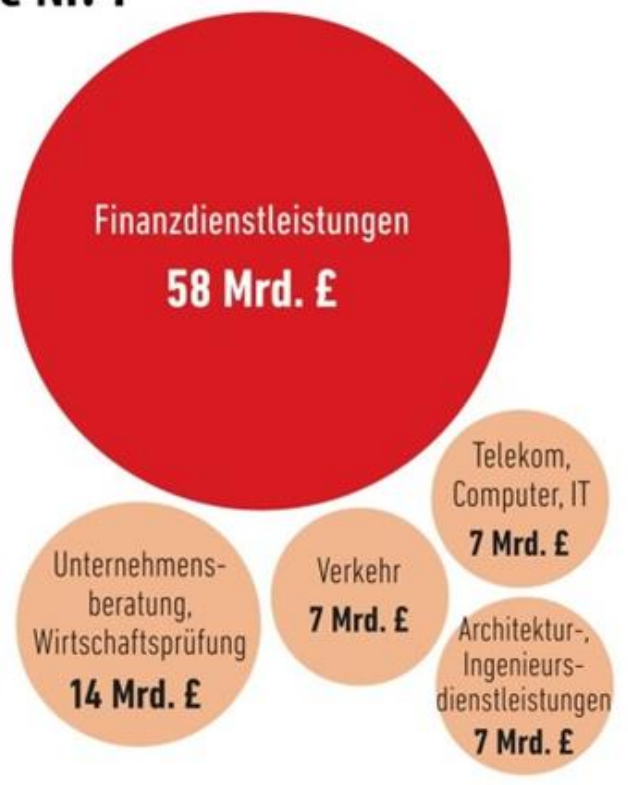


Anhang 4: Jährliches reales Wirtschaftswachstum in der Eurozone, EU27 und Großbritannien

Quelle: Paul J.J. Welfens: BREXIT aus Versehen. Europäische Union zwischen Desintegration und neuer EU. 2. Auflage, Springer Fachmedien Wiesbaden GmbH, 2018, S.559

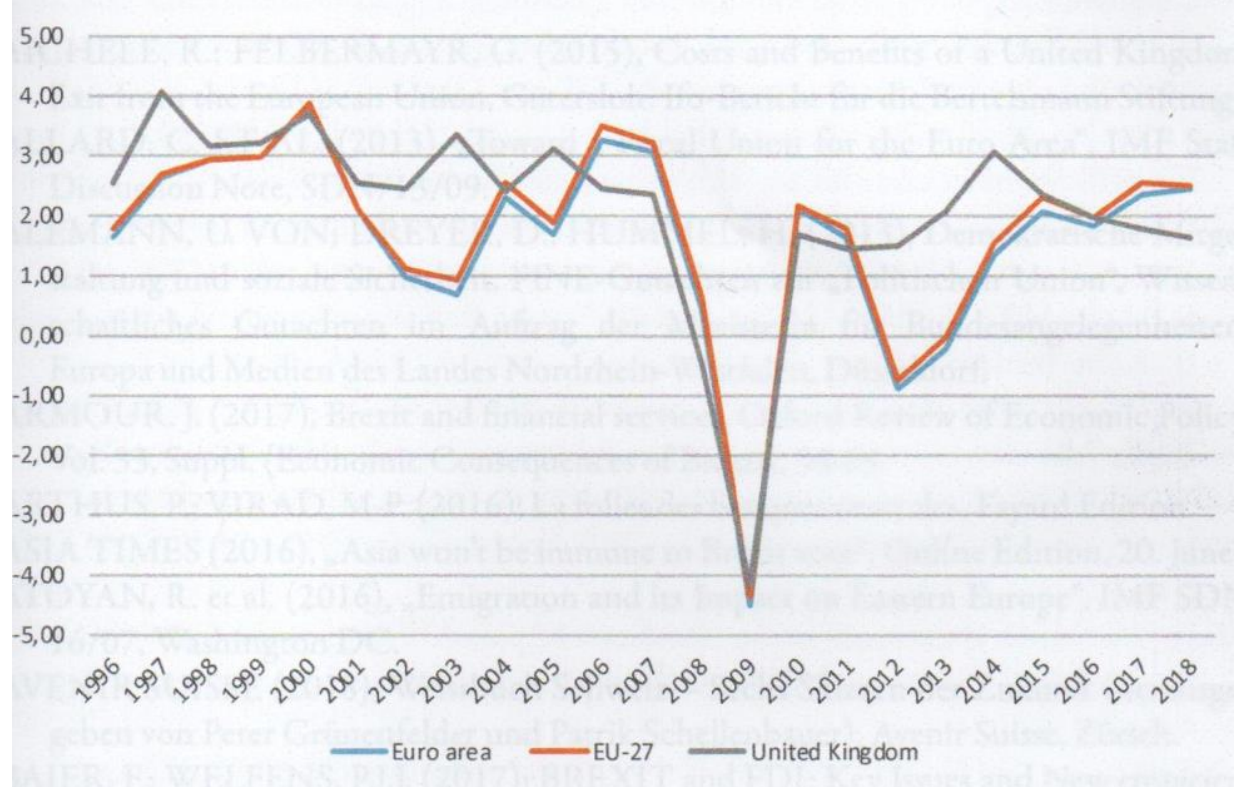

Anhang 5: Anteile der EU-Staaten am Euro-Clearing, April 2016, Angaben in Prozent

Quelle:

https://cdn.static-economist.com/sites/default/files/images/printedition/20170513_FNC657.png 02.02.19, 11:40 Uhr

\section{Britain clears up}

Over-the-counter euro interest-rate derivative turnover, by country, April 2016, \%

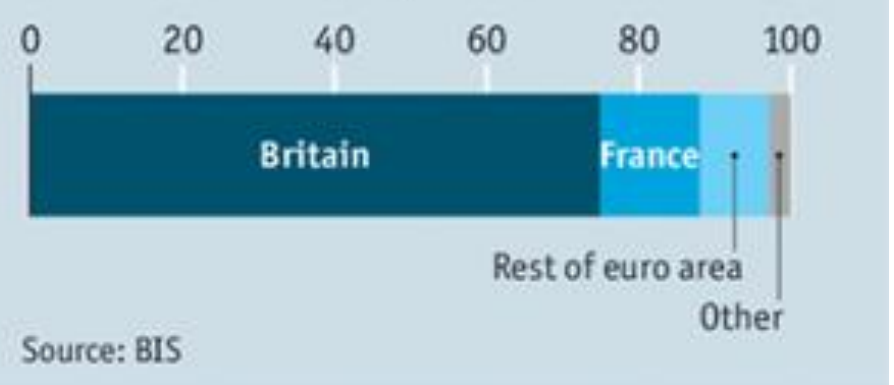

